\title{
Chinese-Russian distance interpreting landscape: a local perspective
}

\author{
Ekaterina Krasnopeyeva ${ }^{1, *}$, Anastasiya Volchkova $^{1}$ \\ ${ }^{1}$ Chelyabinsk State University, Department of Theory and Practice of Translation/Interpreting, 454001129 Kashirinykh St., Russia
}

\begin{abstract}
This paper discusses the Chinese-Russian interpreters' views on the adoption of distance interpreting (DI) technology in the local Russian context. From the intergovernmental to the people-topeople level, the Sino-Russian relationship has been developing as a strategic partnership. Due to travel restrictions and other pandemic containment measures, the demand for virtual communication technology and remote interpreting has been on the rise, which highlighted a variety of implications for the interpreting profession. We present the results of a series of interviews with Chinese-Russian interpreters from Moscow and Chelyabinsk, Russia ( $n=6)$, accompanied by a small-scale online survey $(n=26)$. The exploratory study focuses on the interpreters' experience with technology, and on their perception of DI in general and video remote interpreting in particular. The results suggest that the common DI scenarios in the local ChineseRussian interpreting market (Chelyabinsk region) include video-mediated and over-the-phone consecutive interpreting in business and education settings. The study also reveals an overall positive attitude to the DI technology among the surveyed interpreters, despite such reported challenges as psychological discomfort, connection problems and technical difficulties on the clients' part, which often lead to new responsibilities of an interpreter.
\end{abstract}

\section{Introduction}

While the impact of the COVID-19 pandemic is yet to be fully comprehended, the ongoing changes in everyday practices caused by the containment measures have already become a topic of reflection in many academic and professional communities. Among the major transformations triggered by the restrictions of 2020 is innovation in virtual communication technology. The web-based solutions connecting people via audio and video-link have not only helped protect public health and safety but also ensured the continuity of political, economic and cultural connections at the time of travel bans and lockdowns. The ubiquitous media spaces [1], engendered by audiovisual and computer technologies, have become central to maintaining collaboration and aiding institutional and personal communication.

From the intergovernmental to the people-to-people level, the Sino-Russian relationship has been developing as a strategic partnership. In the unprecedented times of the global pandemic, the traditional forms of cooperation had to be put on hold, and maintaining humanitarian and economic ties in the times of travel restrictions meant resorting to online communication. Ambassador of China to Russia Zhang Hanhui notes that in these unprecedented times "China and Russia maintained mutual understanding, mutual assistance and support, which demonstrated the special and high-level nature of China-Russia relations of strategic cooperation and partnership in the new era" [2] (here and further translation from Russian is done by the authors).

The emergency shift to a digital mode of interaction across the globe has inevitably brought changes to the interpreting domain. While the discussion of the technological turn in interpreting has been gaining momentum in recent years [3-5], the pandemic-inflicted rapid transformation has highlighted a variety of implications that technological mediation may cause both for individuals in the field, and for the development of the profession.

This paper focuses on the interpreters' perception of distance interpreting (DI) practices. It reports on the findings of a small-scale exploratory study combining a series of interviews and a survey of interpreters working in the language pair Chinese-Russian (further we will use "CR interpreting" to refer to interpreting between Chinese and Russian). The structure of this paper is defined by the following research questions:

- RQ1. What DI configurations and communication settings are relevant in the local CR interpreting context?

- RQ2. How do CR interpreters perceive DI and what challenges do they report as the most relevant?

This pilot study is part of the research project on video-mediated interpreting from English, German and Chinese into Russian featuring professional and student consecutive interpreting in simulated typical scenarios of business and technical communication. In view of this, the study also aims to ground the corpus-based

* Corresponding author: y.krasnopeyeva91@csu.ru 
investigation in professional practice by identifying the configurations and communication settings that are in demand in the local Russian CR interpreting context.

\section{Background}

The CR interpreting landscape is defined by the directions of Sino-Russian cooperation. The long-term strategic partnership between China and Russia resulted in a wide range of bilateral initiatives and collaboration in various economic and humanitarian domains.

English has often been used as a lingua franca in Sino-Russian business cooperation, however, as political, economic and cultural ties between the two countries strengthen, more commercial and governmental initiatives require professional $\mathrm{CR}$ interpreting [6]. According to the 2019 China Language Service Industry Development Report, Russian remains one of the top five languages in terms of translation volume in the Chinese language services market [7]. Unofficial estimates show that Chinese takes the third place after English and German in the Russian translation/interpreting market as well [8].

The common jobs performed by a professional CR interpreter in Russia are consecutive interpreting (including such settings as official engagements of government agencies; official business meetings, negotiations; official dinners and receptions; technical meetings and conferences; installation of equipment and staff training; videoconference and telephone interpreting; court interpreting), simultaneous conference interpreting; acting as an expert witness in court hearings; and interpreting or acting as a consulting specialist at the notary public office $[9,10]$.

The onsite interpreting sector of the language services industry has been most heavily affected by the crisis across the globe [11]. The Translation Rating analysis of the 2020 Russian market concludes that the conference interpreting segment was hit the most, as almost all of the events were cancelled due to the restrictions [12]. A survey on the impact of COVID-19 on Language Service Providers in China [13] also showed that onsite interpretation was the most affected by the pandemic $(62.8 \%)$.

The year 2020-2021 was announced the ChinaRussia Year of Scientific and Technological Innovation, involving over a thousand projects and events. In order to maintain crucial links during the pandemic, the development of Internet technology has been made a priority by the two countries. This meant innovation in the spheres of e-commerce, telemedicine, distance teaching and learning, video conferencing, and webbased cultural events [14], many of which required interpreting solutions.

Distance interpreting is a superordinate term used to refer to a variety of technology-mediated interpreting scenarios [4]. These scenarios can be differentiated by a medium of communication (audio/video-mediated interpreting), constellation or configuration (remote/teleconference interpreting), a mode of interpreting (simultaneous/consecutive interpreting), and a communicative setting (conference, community, etc.). Remote interpreting is also often used as an umbrella term to denote "different settings and modalities, for example when all event participants are gathered at one place while the interpreters are located at a different venue, or when the interpreter and one of the interlocutors are both present at the same place" [5]. However, the latter scenario can also be seen as a separate configuration (teleconference interpreting) [4]. Key types of virtual interpreting technology (VIT) [15] include over-the-phone interpreting (OPI) - 电话口译 (diànhuà kǒuyì), video remote interpreting (VRI) 视频口译 (shìpín kǒuyì), and remote simultaneous interpreting (RSI) - 远程同传 (yuănchéng tóngchuán).

The existing body of research on distance interpreting (conducted in different scenarios, including conference and community settings) suggests that technology-mediated solutions can be associated with stress, early onset of fatigue, physical discomfort [16] (RSI), the magnified character of interpreting problems [17] (VRI), reduced feeling of presence [18] (RSI), and ethical issues arising from a need to coordinate the communication as a moderator or a technical specialist [19] (VRI, RSI). In their comprehensive review of survey-based studies of remote interpreting, [20] Corpas Pastor and Gaber conclude that among the common issues associated with remote interpreting are the quality of technical equipment and the discomfort caused by it, as well as a general inconvenience associated with a remote modality. Positive aspects of remote solutions include immediacy, affordability, the reduction of travel costs, and the feeling of privacy and confidentiality. Their own survey of public service interpreters shows the overall positive attitude to remote modality: "each and every one of the positive aspects reaches a higher percentage than any of the negative aspects, except for stress" [20].

As the pandemic unfolded, remote solutions had to be adopted in extremis despite the adverse effect they may have on the dynamics of communication, as well as the psychological and physical wellbeing of an interpreter. The influence of the COVID-19 crisis on the Russian interpreting market is hard to estimate due to the lack of available data. The reports presented at professional conferences, such as Global Dialogue and Translation Forum Russia, as well as discussions in professional online communities, suggest that webbased interpreting scenarios were implemented across the board. The situation with $\mathrm{CR}$ interpreting was discussed during several sessions at the 3rd International Forum of Conference Interpreters "Global Dialogue" in April 2021 (https://ic4ci.com/en/). Talks given as part of "New Reality. Seize the Moment" and "Is There Life Beyond English Interpreting? New Realities. Market Perspectives" sessions show that whereas at the beginning of 2020 the CR interpreting market had been unstable, the need for further cooperation and communication between the governmental agencies, including renewal of agreements and signing of protocols, determined the adoption of videoconferencing that required remote 
interpreting (often performed from the Russian client's office).

Today many interpreters are eager to return back normal and work onsite again. At the same time, it is not unreasonable to assume that hybrid events and DI technology with its cost-effectiveness and immediacy have earned a permanent place in the some of the domains of the interpreting profession. As previous research on DI suggests, the possibility of successful interpreter performance in technology-mediated scenarios is defined by the ability of adaptation [19, 21] In this light, the description of the pandemic and postpandemic status quo of DI in various national, linguocultural and technological contexts can be viewed as an essential step in the development of best practices and interpreter training materials for the new realities of work.

\section{Methods and procedure}

This exploratory study combines a series of interviews with CR interpreters from Moscow and Chelyabinsk, Russia ( $n=6)$, and a small-scale internet-based survey $(n=26)$. As the interviews preceded the survey, the key concepts that emerged in the course of analysis were used in the preparation of the questionnaire. The study focused on the CR interpreters' experience with DI technology, their perception of social, psychological, physiological and ergonomic effects of DI in general, and video remote interpreting in particular.

\subsection{The interviews}

$\mathrm{CR}$ interpreters were recruited for interviews through convenience sampling. The six interviews were conducted between February and August 2021 both face-to-face, via telephone calls and text messaging apps by one of the authors (AV), who is a CR interpreter and an interpreter trainer.

Table 1. Interviewees' profiles.

\begin{tabular}{|c|c|c|}
\hline \multicolumn{2}{|c|}{ Item } & No. \\
\hline \multicolumn{2}{|c|}{ Number of participants } & 6 \\
\hline $\begin{array}{c}\text { Mother } \\
\text { tongue }\end{array}$ & Russian & 6 \\
\hline $\begin{array}{c}\text { Current } \\
\text { place of } \\
\text { residence }\end{array}$ & $\begin{array}{c}\text { Russia } \\
\text { Russia (Moscow) } \\
\text { China }\end{array}$ & 1 \\
\hline Gender & Female & 6 \\
\hline Age & $\begin{array}{c}18-25 \\
26-35\end{array}$ & 1 \\
\hline $\begin{array}{c}\text { Years of } \\
\text { interpreting } \\
\text { experience }\end{array}$ & $\begin{array}{c}3-5 \\
6-10\end{array}$ & 1 \\
\hline
\end{tabular}

\begin{tabular}{|c|c|c|}
\hline $\begin{array}{l}\text { Current } \\
\text { position }\end{array}$ & $\begin{array}{c}\text { Freelance } \\
\text { interpreter } \\
\text { Head of } \\
\text { interpreting } \\
\text { department }\end{array}$ & 2 \\
\hline
\end{tabular}

Table 1 presents an overview of the participants' profiles. All of the interviewees are experienced CR interpreters (3-5 years (1), 6-10 years (1), over 10 years (4)).

The interviewees were provided with a brief description of the project. Each interview started with an open question: "Please describe your experience interpreting via telephone or video-link". The further targeted questions focused on each particular interpreter's experience, but were directed towards the following themes: the interpreters' attitude to an emergency shift to distance interpreting, their perception of remote interpreting; technology-related challenges in DI interpreting, and the common DI settings and platforms used in the CR language pair.

\subsection{The survey}

The online questionnaire was developed based on the interview data, as well as the design of previous surveybased studies (e.g. [20, 22, 23]). In order to explore the CR DI landscape, the list of questions covers a broad range of scenarios and issues, from technical to psychological.

The questionnaire was delivered using Google Forms and also sent out via email (to reach interpreters living in Mainland China). The survey link was distributed in September 2021 through the authors' networks and published in several thematic and professional groups on social networking platforms (VK.com groups "Ya perevodchik" (I am a translator) (72.6K members), groups by Confucius Institute at the Ural Federal University $(2.5 \mathrm{~K})$, Association for Sinology $(2.5 \mathrm{~K})$, and the Faculty of Linguistics and Translation of Chelyabinsk State University (2.7K)). The message accompanying the link to the survey form contained a short description of the project, as well as gave details about the affiliation of the authors.

The questionnaire consisted of 24 multiple choice questions, with five questions aiming at gathering demographic data (age, sex, years of interpreting experience, education, country, etc.). Ten questions were given with an option to fill in a different answer or a comment.

Section 1 of the questionnaire contained a qualifying question "Do you have experience interpreting via video-link or telephone?" As the interviews showed that the meaning of "distance/remote interpreting" as a concept is not clear to some of the interpreters (which is reasonable since the terminology both in the Russian and English languages has not yet been standardized [4]), a short description of interpreting scenarios falling under the umbrella of DI was provided. This question divided the respondents into two groups: the interpreters possessing DI experience (Group 1), and the ones with 
CR interpreting experience, but no DI experience (Group 2).

In Section 2, Group 2 were presented with a set of questions that served the purpose of eliciting attitudes to DI practices and overall perception of DI landscape relevant for $\mathrm{CR}$ interpreters.

Group 1 were additionally asked to reflect on their own DI experience in Section 3. This section contained a list of statements about technology-mediated interpreting and required a participant to mark the statements s/he agreed with, and skip the ones that are not relevant or applicable. The statements were based on the results of the previous studies involving different DI types, such as video-mediated interpreting [19, 24], RSI [16, 23], over-the-phone interpreting [21], and their effect on physiological and psychological, ergonomic, social, and cognitive aspects of interpreting.

At the time of paper preparation, 26 self-selected interpreters took part in the survey, male and female, of different ages and backgrounds, including participants from Russia (23), Germany (1), Spain (1), and China (1). As an overview in [20] shows, the studies surveying interpreters specifically tend to receive very few responses. Considering that $\mathrm{CR}$ is not the most common language combination for many locations in Russia (cf. the argument in [9] stating the regional specificity of Russian CR interpreting market), these numbers were deemed sufficient for an exploratory pilot study.

Table 2. Survey participants' profiles.

\begin{tabular}{|c|c|c|}
\hline \multicolumn{2}{|c|}{ Item } & No. \\
\hline \multicolumn{2}{|c|}{ Number of participants } & 26 \\
\hline \multirow{2}{*}{ Mother tongue } & Russian & 25 \\
& Chinese & 1 \\
\hline \multirow{3}{*}{ Current place of } & Russia & 23 \\
residence & China & 1 \\
& Germany & 1 \\
\multirow{2}{*}{ Gender } & Spain & 1 \\
\hline \multirow{3}{*}{ Age } & Female & 23 \\
& Male & 3 \\
\hline \multirow{2}{*}{ Years of } & $18-25$ & 5 \\
interpreting & $26-35$ & 14 \\
experience & $36-45$ & 4 \\
& no answer & 3 \\
\hline \multirow{2}{*}{ DI experience } & $<1$ & 9 \\
& $6-10$ & 7 \\
& $>10$ & 6 \\
\hline
\end{tabular}

The sample included 10 participants with DI experience (Group 1). Another 16 reported having onsite interpreting experience (consecutive or simultaneous) (Group 2). Group 1 included female (9) and male (1) interpreters in the age groups 25-34 (7) and 35-44 (3) from Russia (8), China (1) and Germany (1). Their mother tongue was Russian (10), and working languages included Chinese (10) and English (8). Out of ten, four participants had over 10 years of interpreting experience, three have worked for 5-10 years, and the other three had 1-5 years of experience. The interpreters have worked face-to-face in the following settings: healthcare (1), administrative (2), business (6), education (6), IT and engineering (1). Compared to Group 2, Group 1 consisted of more experienced interpreters.

Most of the participants in both groups have a degree related to translation/interpreting or language education (Specialist degree -8 , Bachelors' degree -6 , Continued professional education programme - 6 , Doctoral degree -1). Only five of the participants had DI training (including professional development courses and self-education in OPI, RSI and VRI).

\section{Results and discussion}

This section combines the key findings that emerged in the course of the study. It starts with the relevant DI types and settings common for the $\mathrm{CR}$ language combination in the local context. Among the topics discussed below are also the CR interpreters' DI experience and their perceptions.

\subsection{DI settings and configurations in the local context}

All the interviewed interpreters at some point in their career have interpreted via either audio or video-link, or have organised events that required remote interpreting. Although none of them have had an extensive DI experience.

Table 3 presents the replies to the survey question about the interpreting experience in several DI scenarios (Group 1). While we can see that the responses vary, when combined with the interview results, over-thephone interpreting and videoconference interpreting emerge as the most relevant configurations for the local context.

Table 3. Responses to the question "How often do you interpret in the following scenarios?" (Group 1).

\begin{tabular}{|c|c|}
\hline Configuration & $\begin{array}{c}\text { Number of } \\
\text { responses }\end{array}$ \\
\hline Over-the-phone interpreting & $\begin{array}{c}\text { Very often (2) } \\
\text { Sometimes (2) } \\
\text { Rare (6) }\end{array}$ \\
\hline $\begin{array}{c}\text { Videoconference interpreting } \\
\text { (consecutive, chuchotage) } \\
\text { (Skype, Zoom etc.). Interpreter is } \\
\text { on-site }\end{array}$ & $\begin{array}{c}\text { Very often (3) } \\
\text { Sometimes (2) } \\
\text { Rare (2) }\end{array}$ \\
\hline $\begin{array}{c}\text { Rever (3) } \\
\text { during small videoconferences } \\
\text { (Skype, Zoom, Teams, etc.). } \\
\text { Interpreter is remote / all of the } \\
\text { participants are remote }\end{array}$ & $\begin{array}{c}\text { Very often (2) } \\
\text { Sometimes (1) } \\
\text { Rare (2) }\end{array}$ \\
\hline Monologue RSI (e.g. webinar) \\
(Zoom and RSI platforms) & Often (1) \\
& Rometimes (1) \\
\hline Dialogue RSI (e.g. doctor-patient \\
communication) & Nery often (5) \\
\hline (Zoom and RSI platforms) & Sometimes (1) \\
Rare (2) \\
Never (6)
\end{tabular}


When asked to estimate the demand for CR videomediated interpreting on the scale from 0 to 5 , survey participants in Group 1 answered that it is very high (5), high (2), moderate (1) and low (2). Most of the Group 2 participants who have not worked via video-link estimated the demand as moderate (13) or high (3). Both groups agreed that among the common communicative settings for CR DI are business (22), education (10), politics and diplomacy (10), healthcare (5), culture (5), and administrative settings (5).

In terms of platforms, the interpreters have worked with Zoom (8), WeChat (7), Skype (6), Microsoft Teams (2), and Cisco WebEx (1).

Five interviewed interpreters shared their experience interpreting business meetings held via a China-based app WeChat app (https://www.wechat.com/). WeChat is marketed as a multi-faceted platform. It combines chat and video calls features with a variety of services including newsfeed, games, local services, online payment feature, and is a go-to platform for most Chinese users. Since the app is not common in Russia, when the Chinese side requests a meeting via WeChat, an interpreter may act as an expert user. P4 noted that sometimes in b2b negotiations via WeChat the Russian side prefers to facilitate communication by giving an interpreter more freedom to discuss the issues at hand and report a summary of the discussion.

While OPI and VRI can be performed using video chat (among the popular apps in China are WeChat, QQ, 527meeting, Dingding [25]) and video conferencing apps (Zoom Meeting, Zhumu, Kedacom, Wanghui Technology, Huijian, Youting, Huawang, Pugongying, OM, Boshi [26]), RSI requires specialized platforms that rely on cloud-based technology and highquality video transmission. RSI solutions are on the rise and are gradually being considered by international institutions (cf. [27]). However "Live streaming into Mainland China is another major issue with most RSI platforms as there are no servers allocated at all in China from the platforms based outside China" [28]. Therefore, international online conferences involving participants from Mainland China may either require RSI features offered by non-specialized platforms, such as Zoom, or service providers targeting the region (cf. "The TRANSLIT RSI platform also uniquely supports mainland China with no extra set-up" (https://rsi.translit.com/), or China-based solutions, such as Akkadu (https://www.akkadu.com/).

Zoom and Skype are also mentioned by the interviewed interpreters as apps Chinese counterparts prefer for connecting with foreign partners. In $b 2 b$ meetings, in many cases the platform for communication is chosen by the Chinese side, and the Russian partners adapt to the preferences. As P5 notes, "If the conference participants have no connection problems, everything goes as planned. However, the conferences often get rescheduled, which is quite inconvenient".

The interpreters admit that in $\mathrm{b} 2 \mathrm{~b}$ negotiations, the technology-mediated setting has transformed the dynamics of communication. "Traditional protocol has been replaced by written email communication. Videomediated meetings are much shorter than face-to-face ones. Only essential issues are discussed" (P5). When it comes to building business and personal connections with Chinese counterparts, many of the valued aspects of face-to-face encounters simply cannot be replaced by videoconferencing technology. The high-context nature of Russian and Chinese cultures influences language mediation.

The possibility of differences in acceptance of video-mediated communication and interpreting in different cultures has been pointed out in literature (cf. legal video-mediated interpreting [24]). The lack of paralinguistic cues has been identified as a key challenge of DI; however its socio-cultural implications have not been widely explored. Therefore, the use of video-mediated interpreting in China-Russia business settings requires further investigation from the point of view of socio-cultural norms and etiquette.

\subsection{The CR interpreters' perception of DI}

The following list of characteristics was presented to all the survey participants, irrespective of their DI experience, in order to elicit their perception of working in a technology-mediated environment.

An interpreter when working via video-link...

- Experiences psychological discomfort. (12)

- Easily adapts to the new conditions, including techrelated challenges. $(8)$

- Takes on the role of a technical specialist. (6)

- Feels alienated, isolated. (6)

- Takes on the role of a technical specialist. (6)

- Gets tired faster. (6)

- Is productive. (2)

- Feels safe. (2)

- Feels energized, motivated. (1)

- Experiences physical discomfort. (1)

- * Is overloaded with unnecessary information, has to perform the tasks that should be done by other specialists. (1)

- * Independent and level. (1)

- * ...Every interpreter is different. (1)

- Has difficulty concentrating. (0)

- Is mentally exhausted. (0)

(*The statements added to the list by the participants.)

We can see that the most respondents assume that video-mediated interpreting may have psychological effects. Interestingly, only two of the 12 respondents who agreed with the statement have worked via videolink. Experienced interpreters (Group 1) relate the most to the statements referencing adaptability of an interpreter and the need to "perform the tasks that should be done by other specialists". While the choice of a platform may be out of interpreters' hands, the survey showed that when it comes to technologymediated communication, the interpreter often has to be tech-savvy as s/he has to take on a role of a technical specialist, or, as one of the participants puts it, the 
interpreter is "overloaded with unnecessary information".

The interpreters in Group 1 were additionally asked to mark the statements that reflect their experience of remote consecutive (CI, fulfilled by nine participants) or remote simultaneous interpreting (SI, fulfilled by four participants). In terms of organisational and technical aspects, the interpreters report that DI is always associated with technical difficulties, i.e. bad connection $(\mathrm{CI}-4)$ that they have to perform the job of a moderator (CI -5 ; SI - 2), and that the speakers tend to interrupt each other (CI, 5), which also causes difficulty for the interpreter.

When characterizing their experience, they agreed that it is harder to work when they do not have a view / have a limited view of the facial expression and gestures of the speaker $(\mathrm{CI}-6$; SI -2$)$ and that it is harder to grasp the emotional component of the speech $(\mathrm{CI}-3$; SI $-1)$. Other statements the interpreters agreed with or added to the list were: a more stressful nature of remote interpreting (CI - 2; SI - 1), technical difficulties on the clients part (CI, 1; SI, 2), an interpreter getting tired earlier (CI - 1; SI, -2), cultural differences of the parties getting more apparent in the remote setting $(\mathrm{CI}-1)$, and peculiar lexical difficulties, including generational differences $(\mathrm{CI}-1)$.

The interviews reflect mostly positive experiences of working in distance mode, but underline that "The experience depends a lot on the quality of connection" (P5), and that "It is important to check all the technical aspects beforehand" (P1).

P6, head of an interpreting department, mentions that the CR interpreters who she works with have remote interpreting experience and have never reported any technical difficulties. This however may be connected to the fact that the company she works for provides a technical team for the organisation of online events.

When elaborating on DI practices, the interviewed experienced interpreters (P1, P3) also focus on psychological issues related to a distance mode: 'Psychologically, it is challenging to experience being in one place knowing that a person whom you are interpreting somewhere else,' shares P3 who has worked in simultaneous and consecutive modes for over 10 years. She also describes a feeling of alienation. The lack of visual component of communication in DI deprives the interpreter of the ability to perceive the emotions of the speaker. P1, who is an experienced interpreter working mostly face-to-face in consecutive mode, admits that she feels a distance between the speakers when interpreting via video-link. "Let's be objective, it is harder to perceive what a person is saying via video compared to face-to-face," she notes.

Despite the known technological, psychological and ergonomic issues associated with DI, both the interviews and the survey paint a positive view of DI adoption in extremis. In Group 1, seven out of ten experienced interpreters express a positive attitude to the use of video-mediated solutions; and three participants elaborate on their realistic view of the situation: "This is a good alternative when there is no other choice." "It is ok for business negotiations, but may cause great problems when used for technical interpreting." "There are pros and cons". "This is an objective reality, so my position is neutral'. In Group 2, 13 participants share a positive attitude to DI, one remains neutral ("I would rather consider it a necessity which we have to adapt to") and two perceive DI adoption negatively: "Problems with internet connection are common for the region where I live. This makes any video-mediated communication almost impossible, especially when you need to comprehend what is being said".

\section{Conclusion}

The restrictions imposed by the nations in an effort to contain the coronavirus pandemic have facilitated the adoption of virtual communication technologies, including DI solutions. Web-based communication has become an essential tool for ensuring continuity of the Sino-Russian strategic partnership on a variety of levels during the time when face-to-face communication is a rare commodity.

This paper discussed the results of a small-scale exploratory study aiming to grasp the Chinese-Russian interpreters' perceptions and experiences of technologymediated interpreting. The results of the survey accompanied by a series of interviews suggest that while the majority of the participants admit a high demand for distance interpreting, many of them have limited experience working in the DI scenarios. Although due to a small sample size we cannot generalise the findings to the $\mathrm{CR}$ interpreting market, it is possible to say that in the local Chelyabinsk CR interpreting market the most common scenario is consecutive (dialogue) video/audio-mediated interpreting in business and technical domains.

Despite the fact that video-mediated interpreting is perceived by the surveyed interpreters as an activity associated with technical difficulties on the clients' part, and causing psychological discomfort on the interpreter's part, the overall positive or neutral attitude to the new modality prevails. The participants agree that an interpreter can easily adapt to the new conditions, including the technology-related challenges and performing the job of a technician or a moderator. As one of the interpreters notes, "This [video-mediated interpreting] is a good alternative when you have no other choice".

The study also illustrated the need for a sociocultural perspective in DI research. Since technological mediation influences the dynamics of communication, the resulting changes can be perceived differently by the speakers of different cultural backgrounds.

To the best of our knowledge, CR technologymediated interpreting has not been widely described as a practice and is yet to receive attention from both Chinese and Russian interpreting scholars. Therefore, despite a number of limitations, including the subjective nature of the methods and the small sample size, this study contributes to the conversation on the 
technological changes in the Chinese-Russian interpreting landscape.

The authors would like to thank the participants of the study for their time and invaluable input.

This research is supported by the President's Grant for the State Support of the Young Russian Candidates of Sciences (Project No. MK-73.2021.2).

\section{References}

1. S. Harrison, Media Space 20+ Years of Mediated Life, 9-16 (Springer, London, 2009)

2. Chinese Embassy in Russia. Ambassador Zhang Hanhui Interview. Retrieved from: http://ru.chinaembassy.org/rus/sghd/t1842946.htm (2020) (In Russian)

3. O. Jiménez Serrano, Revista Tradumàtica. Tecnologies de la Traducció, 17, 20-31(2019)

4. S. Braun, Routledge Handbook of Translation and Technology, 271-288 (Routledge, London, 2019)

5. C. Fantinuoli, Interpreting and Technology, 1-12 (Lang. Sci. Press, Berlin, 2018).

6. N. N. Voropayev, Translator: Sci. Lit. J., 14, 180 198 (2014). (In Russian)

7. China Language Service Industry Development Report (Translators Association of China, 2019)

8. PrimaVista Translation Agency. Most in-demand languages in the Russian translation market. Retrieved from: https://www.primavista.ru/blog/2016/09/15/perspek tivnyie-yazyiki (2016)

9. N. N. Voropaev, On the challenges of becoming a Chinese interpreter, in Proceedings of an Int. Sci. Conf. "Chinese: Linguistic and Methodological Aspects", 130-135. Retrieved from: https://www.elibrary.ru/item.asp?id=27623186 (2016) (In Russian)

10. N. N. Voropaev, Translator: Sci. Lit. J., 19, 187198. Retrieved from: https://www.elibrary.ru/item.asp?id=41170581(201 9). (In Russian)

11. Nimdzi Insights. Interpreting in times of COVID19. Retrieved from: https://www.nimdzi.com/interpreting-in-times-ofcovid-19/ (2020)

12. Translation Rating. Volume and dynamics of the Russian translation market in 2020. Retrieved from: https://translationrating.ru/market-size-2020/ (2020) (In Russian)

13. L. Wang, M. Yongye, S. Jiangwei, R. Jie, L. Ying, X. Yushang, W. Xiaomeng, Z. Zilong, Z. Yuanxia, A Survey Report on the Impact of COVID-19 on Language Service Providers in China. Retrieved from: https://slator.com/china-releases-survey-onhow-the-coronavirus-is-affecting-the-languageindustry/ (2020)
14. Russian International Affairs Council. RussiaChina Dialogue: model 2020: report No. 58/2020. 中俄对话: 2020模式. Retrieved from: russiancouncil.ru/report58 (2020) (In Russian, Chinese)

15. Nimdzi Insights. The virtual interpreting landscape. Retrieved from: https://www.nimdzi.com/virtualinterpreting-landscape/ (2020)

16. B. Moser-Mercer, Meta: j. des trad. / Meta: Tran. J, 50 (2), 727-738 (2005)

17. S. Braun, J. L. Taylor, Videoconference and remote interpreting in criminal proceedings, 145-185, (Intersentia Publishing Ltd., Cambridge, 2012)

18. P. Mouzourakis, Interpreting. 8 (1), 45-66 (2005)

19. S. Braun, Interpreting. 9 (1), 21-46 (2007)

20. G. Corpas Pastor, M. Gaber, SKASE J. of Tr. and Int., 13 (2), 58-78 (2020)

21. L. Ko, Ling. Antverp., Themes in Tran. Stud., 5, 325-338 (2006)

22. M. Albl-Mikasa, M. Eingrieber, FITISPos Int. J., 5(1), 33-44 (2018)

23. K. G. Seeber, Interdependenzen und Innovationen in Translation und Fachkommunikation, 123-172, (Frank \& Timme, Berlin, 2020)

24. S. Braun, Videoconference and remote interpreting in criminal proceedings, 265-287, (University of Surrey, Guildford, 2011)

25. 2020 年永久免费视频会议软件排名有哪.

Retrieved

from: https://www.sohu.com/a/393534015_403675 (2020)

26. 2020 视频会议热门软件排行榜十大视频会议软 件介绍. Retrieved from: https://www.jiaochengzhijia.com/android/news/112 68.html (2020)

27. European Commission Directorate-General for Interpretation (SCIC). Report "Interpreting Platforms. Consolidated test results and analysis" Retrieved from: https://ec.europa.eu/education/knowledge-centreinterpretation/ (2021).

28. B. Song, Considerations When Using Remote Simultaneous Interpreting During the Pandemics. Retrieved from: https://www.bernardsong.com/post/considerationswhen-using-remote-simultaneous-interpretingduring-the-pandemics (2020) 